


\title{
GEOLOGIA, GEOCRONOLOGIA E GEOQUÍMICA DO GRANITO DE INCHOPE (MOÇAMBIQUE)
}

\section{GEOLOGY, GEOCHRONOLOGY AND GEOCHEMISTRY OF THE INCHOPE GRANITE (MOZAMBIQUE)}

\author{
V. A. Manjate ${ }^{1} \&$ C. C. G. Tassinari ${ }^{2}$
}

\begin{abstract}
Resumo - A região de Gondola-Nhamatanda localiza-se na parte limítrofe entre as províncias de Manica e Sofala, centro de Moçambique. Ela está geologicamente inserida no complexo de Bárue (Grupo de Chimoio). O Complexo de Bárue é composto pelos grupos Supracrustais de Macossa e Chimoio intrudidos por rochas plutônicas de várias composiçôes. As amostras de rochas da área de trabalho foram estudadas em termos de petrografia; geoquímica de elementos maiores e traço; geocronologia e geoquímica isotópica pelos métodos $\mathrm{U}-\mathrm{Pb}$ em zircão, $\mathrm{Rb}-\mathrm{Sr}$ e $\mathrm{Sm}-\mathrm{Nd}$ em rocha total e $\mathrm{Rb}-\mathrm{Sr}$ em minerais para o Granito de Inchope. Este estudo permitiu determinar os litotipos que intrudiram o Grupo Supracrustal de Chimoio, suas idades de cristalização e de diferenciação dos magmas que formaram seus protólitos e ainda, o enquadramento tectônico. As rochas estudadas são basicamente calci-alcalinas peraluminosas. Elas cristalizaram no Mesoproterozoico (idades $\mathrm{U}-\mathrm{Pb}$ e $\mathrm{Rb}$ - $\mathrm{Sr}$ ) a partir de protólitos Paleoproterozoicos $\left(\mathrm{T}_{\mathrm{DM}}=2,4-2,3\right.$ $\mathrm{Ga}$ ). Os dados isotópicos sugerem que estas rochas foram geradas por fusão parcial que provavelmente envolveu mistura (valores negativos de $\varepsilon_{\mathrm{Nd}}$ ) da crusta Arcaica/Paleoproterozoica e magma mesoproterozoico a $1100 \mathrm{Ma}$ e sofreu retrabalhamento durante a aglutinação do Gondwana (Orogenia Pan-Africana).
\end{abstract}

Palavras-chave - Orogênese; Geocronologia; Idade modelo; Idade isocrônica; Cristalização

Abstract - The Gondola-Nhamatanda region is located at the border between the provinces of Manica and Sofala, central part of Mozambique. It is geologically inserted in the Bárue Complex (Chimoio Group). The Bárue complex is composed of the Macossa and Chimoio supracrustal groups intruded by plutonic rocks of various compositions. The rocks samples of the work area were studied in terms of petrography; geochemistry of major and trace elements; geochronology

\footnotetext{
1 Direcção Nacional de Geologia, Praça 25 de Junho, 217 - Maputo, Moçambique; vmanjate@yahoo.com.br

2 Instituto de Geociências/ USP, Rua do Lago, 562, Cidade Universitária, São Paulo, Brasil; ccgtassi@usp.br
} 
and isotope geochemistry by the follow methods: U-Pb in zircon, Rb-Sr and Sm-Nd in whole rock, and Rb-Sr in minerals for the Inchope granite. This study allowed determining the lithotypes that intruded the Chimoio Supracrustal Group, the crystallization and differentiation ages of the magmas that formed the protoliths, and the tectonic framework. The studied rocks basically are calc-alkaline and peraluminous. They have been crystallized in the Mesoproterozoic $(U-P b$ and $R b$-Sr ages) from Paleoproterozoic protoliths $\left(T_{D M}=2.4-2.4 G a\right)$. The isotopic data suggest that they have been generated by partial melting that probably involved the mixture $\left(\varepsilon_{N d}\right.$ negative values) of Archean/Paleoproterozoic crust and Mesoproterozoic magma at $1100 \mathrm{Ma}$, and suffered reworking during the Gondwana amalgamation (Pan-African Orogeny).

\section{Keywords - Orogenesis; Geochronology; Model age; Isochron age; Crystallization}

\section{1 - Introduçáo}

O Granito de Inchope faz parte do grupo de rochas plutônicas que intrudiram o Grupo Supracrustal de Chimoio no Mesoproterozoico (GTK CONSORTIUM, 2006). Este granito aflora no Posto Administrativo de Inchope (Fig. 1), Distrito de Gondola, em Moçambique, e contém veios pegmatíticos com mineralizações de cassiterite e columbite (LKAB, 1980; LACHELT, 2004). Na parte Este é intrudido pelo carbonatito do Monte Xiluvo (LACHELT, 2004; MELLUSO et al., 2004; GTK CONSORTIUM, 2006) e está em contacto tectônico com rochas do Karroo vulcânico, que MANNINEN et al. (2008) atribuiram a flexura Nuanetsi-Sabi (NNE-SSW), originada por magmatismo relacionado com plumas datadas de 183 Ma e subsequente fraturamento do Gondwana durante o Jurássico Inferior-Médio, que consiste principalmente de fluxos de lava toleítica a picrítica, rochas piroclásticas félsicas, bem como veios e diques relacionados com o fraturamento do Gondwana e a abertura do Oceano Índico.

O Granito de Inchope é abordado em trabalhos de mapeamento geológico a escala 1:250.000 (GTK CONSORTIUM, 2006) e de prospecção e pesquisa geoquímica (LKAB, 1980; KORKIAKOSKI, 2007). Com os dados da pesquisa foi possível determinar as idades de cristalizaçáo, retrabalhamento e modelo do magma que formou o protólito do Granito de Inchope, bem como os seus parâmetros petrogenéticos e enquadramento tectônico.

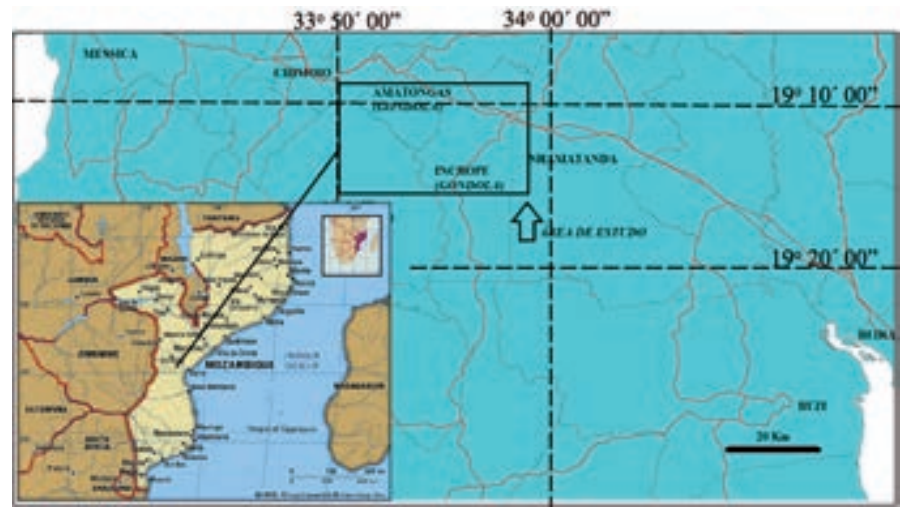

Fig. 1 - Mapa de localização da área de estudo, parte central de Moçambique. As linhas vermelhas são estradas e as escuras limites geográficos de Postos Administrativos (adaptado de http://www.cenacarta.com/modules.php?name=Downloads\&d_op=getit\&lid=6) 


\section{2 - Enquadramento geológico}

A área de estudo localiza-se no Complexo de Bárue (Grupo de Chimoio). PINNA et al. (1987), no mapa geológico a escala 1:1.000.000, apresentam este complexo dividido em cinco unidades litoestratigráficas, nomeadamente os grupos de Nhamatanda, Madzuire, Changara, Canxixe e Matambo. Estes grupos não podem ser identificados como entidades separadas nas imagens radiométricas e aeromagnéticas. De acordo com LACHELT (2004), relativamente ao Cráton de Kalahari, o Complexo de Bárue é limitado pelos grupos de Gairezi e Umkondo, pertencentes ao Ciclo Orogénico Irumide. PINNA et al. (1987) atribuem idades de $1100 \mathrm{a} 800 \mathrm{Ma}$ às principais rochas do Complexo de Bárue.

O GTK CONSORTIUM (2006) apresenta um mapa geológico no qual não mantém os grupos propostos anteriormente. O Complexo de Bárue (Fig. 2) é dividido nos grupos supracrustais de Macossa e Chimoio, ambos intrudidos por rochas plutônicas mesoproterozoicas de várias composiçôes retrabalhadas no Neoproterozoico. Nesta divisão, o Complexo de Bárue é limitado a Este por uma série de falhas do Rifte Este Africano e formaçôes mesozoicas em parte cobertas por sedimentos do quaternário, a Oeste por um cizalhamento esquerdo ao longo da margem cratónica Arcaica, a Norte por um cavalgamento orientado para Norte e a Sul, as rochas do complexo se mostram cobertas por camadas do Fanerozoico.

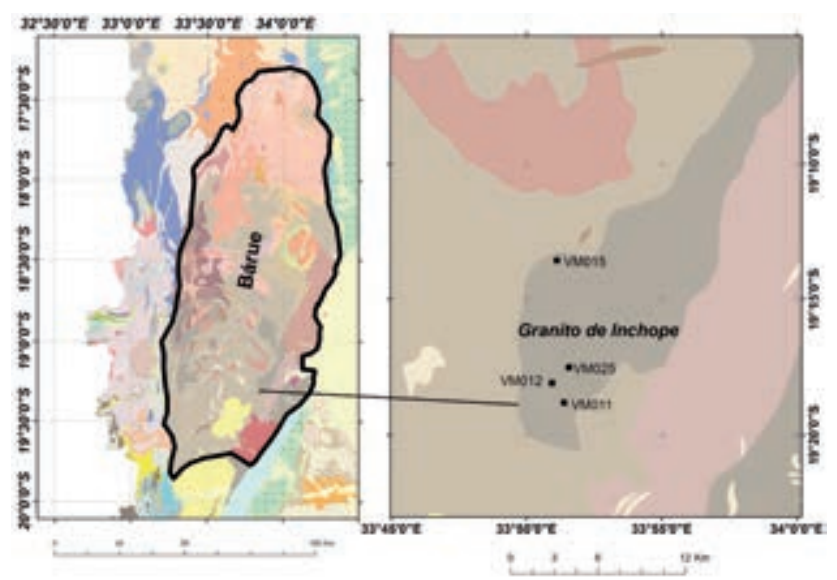

Fig. 2 - Mapa de localização do Complexo de Bárue (adaptado de GTK CONSORTIUM, 2006).

\section{3 - Materiais e métodos}

Para este estudo foram coletadas um total de quatro (4) amostras de Granito de Inchope. $\mathrm{Na}$ amostragem foi obedecido o critério de selecionar material sem alteração, com tamanho 6 a 10 vezes maior que o do maior cristal e com peso aproximado de $3 \mathrm{~kg}$. Destas amostras, quatro (4) foram submetidas a estudos petrográficos usando lâminas delgadas; duas (2) à geoquímica de elementos maiores, traço e terras raras; duas (2) a estudos geocronológicos e quatro (4) à geoquímica isotópica no Laboratório do Instituto de Geociências da Universidade de São Paulo (Brasil). 
Duas (2) amostras de granito foram pulverizadas no moinho de ágata e analisadas por fluorescência de raios-X, usando pastilha fundida para elementos maiores $\left(\mathrm{SiO}_{2}, \mathrm{TiO}_{2}\right.$, $\mathrm{Al}_{2} \mathrm{O}_{3}, \mathrm{Fe}_{2} \mathrm{O}_{3}, \mathrm{MnO}, \mathrm{MgO}, \mathrm{CaO}, \mathrm{Na}_{2} \mathrm{O}, \mathrm{K}_{2} \mathrm{O}$ e $\mathrm{P}_{2} \mathrm{O}_{3}$ ) e ICP-MS, e pastilha prensada para elementos traço e terras raras.

Foram analisadas duas (2) amostras deste granito pelo método U-Pb usando $L A-I C P$ $M S$ em grãos de zircão, tendo como referência as imagens de catodoluminiscência. Estas amostras foram, em primeiro lugar, pulverizadas usando moinho de disco. A porção rica em minerais pesados foi tratada com bromofórmio e o concentrado de minerais pesados foi processado no separador electromagnético $F R A N T Z$ a $0,5 \mathrm{~A}$. A fração não magnética foi tratada com Iodeto de Metil e a fração que contém minerais pesados foi, mais uma vez, processada no separador electromagnético FRANTZ a 1,0 e 1,5A, respectivamente. A purificação final da fração de zircôes foi feita com selecção a pinça no microscópio binocular.

As concentraçóes de Rb e Sr das quatro (4) a mostras de granito foram obtidas por fluorescência de raios-X. A separaçáo de $S r$ foi feita pela técnica convencional de troca catiônica em colunas de resina AG50WX8, após dissolução com $\mathrm{HF}-\mathrm{HNO}_{3}-\mathrm{HClO}_{4}$. O erro para a razão isotópica ${ }^{87} \mathrm{Rb} /{ }^{86} \mathrm{Sr}$ foi calculado a partir da propagação de erros analíticos das variáveis usadas na equação. As razóes isotópicas ${ }^{87} \mathrm{Sr} /{ }^{86} \mathrm{Sr}$ foram determinadas por espectrometria de massa de fonte sólida, num sistema de ultra-alto vácuo de $10^{-8} \mathrm{mBar}$, e voltagem de aceleração de $10 \mathrm{KV}$, usando filamentos de Ta e tensão de $8000 \mathrm{~V}$. Os erros $(2 \sigma)$ variaram de $0,000066-0,000119$ para $\mathrm{Sr}$ e foram normalizadas para o valor ${ }^{86} \mathrm{Sr} /{ }^{88} \mathrm{Sr}$ = 0,1194 segundo as recomendaçóes de DE PAOLO (1981). O Valor médio para a razão ${ }^{87} \mathrm{Sr} /{ }^{86} \mathrm{Sr}$ do padrão NBS-987 de Janeiro a Junho/2011 = 0,710265 $\pm 0,000031$. As concentraçôes de $\mathrm{Sm}$ e $\mathrm{Nd}$ foram obtidas pela técnica convencional de troca catiônica em colunas de resina AG50WX8, após dissolução com $\mathrm{HF}^{-} \mathrm{HNO}_{3}-\mathrm{HClO}_{4}$.

As razóes isotópicas ${ }^{143} \mathrm{Nd} /{ }^{144} \mathrm{Nd}$ (medidas como $\mathrm{Nd}^{+}$) das quatro (4) amostras de granito foram determinadas por espectrometria de massa de fonte sólida, num sistema ultra-alto vácuo de $10^{-8} \mathrm{mBar}$ e voltagem de aceleração de $10 \mathrm{kV}$, usando filamento de Ta-Re e tensão de $10000 \mathrm{~V}$, normalizadas para o valor de ${ }^{146} \mathrm{Nd} /{ }^{144} \mathrm{Nd}=0,7219$, segundo as recomendaçôes de DE PAOLO (1981).

\section{4 - Resultados}

\section{1 - Petrografia}

O Granito de Inchope é uma rocha de coloração acastanhada, porfirítica e de granulação variável entre fina a média. A rocha apresenta textura granular xenomórfica a hipidiomórfica. Os minerais principais observados são o quartzo $(-30 \%)$, plagioclase $(-10 \%)$, microclina $(-40 \%)$, Biotite e muscovite, e como acessórios os minerais opacos, titanite, anfíbola (hornblenda), zircão, apatite, monazite e silimanite. Pode-se observar ainda a mirmequita. $\mathrm{O}$ quartzo ocorre em grãos incolores, anédricos, com limites que frequentemente se adaptam às formas dos outros minerais e apresenta extinção ondulada bem como desenvolvimento de subgrãos. A plagioclase existe sob forma de cristais subédricos à anédricos com lamelas de deformação e extinção ondulada, mostrando-se por vezes alterado localmente para sericita. A microclina apresenta pertitas (Fig. 3). 
A Biotite se apresenta sob grãos de forma subédrica, com pleocroísmo típico entre castanho claro a castanho escuro, mostrando por vezes geminaçôes. Os minerais opacos se apresentam preenchendo espaços na muscovite. O zircáo ocorre preenchendo espaços entre a Biotite e a muscovite.
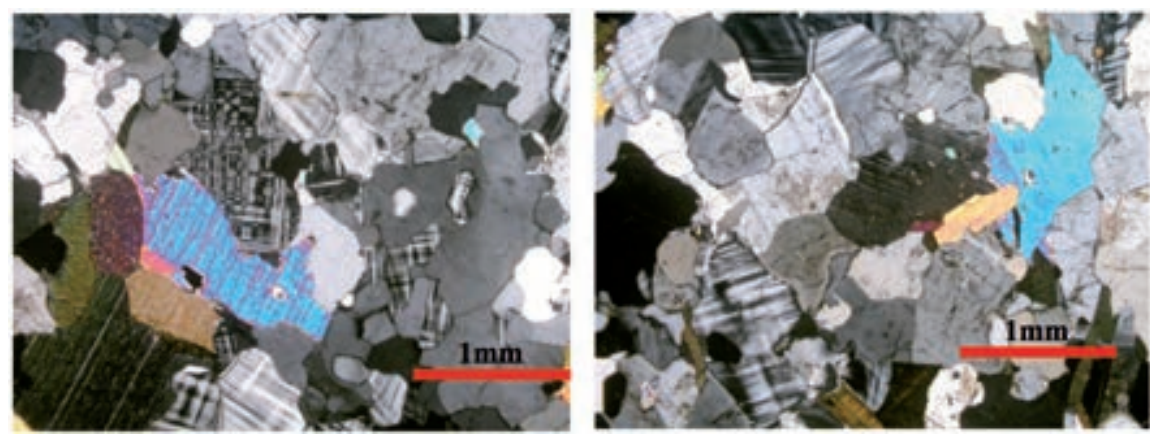

Fig. 3 - Fotomicrografia da amostra VM015 (S19 13'35,5”/E33 51'12,5”) mostrando microclina, biotite, muscovite e plagioclase. Polarizadores cruzados.

\section{2 - Litogeoquímica}

As rochas de Inchope têm um carácter ácido e classificam-se em granitos e plotam na série cálcio-alcalina no diagrama AFM de IRVINE \& BARAGAR (1971). Os granitóides de Inchope apresentam um carácter peraluminoso no diagrama de SHAND (1943), conforme a Fig. 4 e os dados da tabela 1.
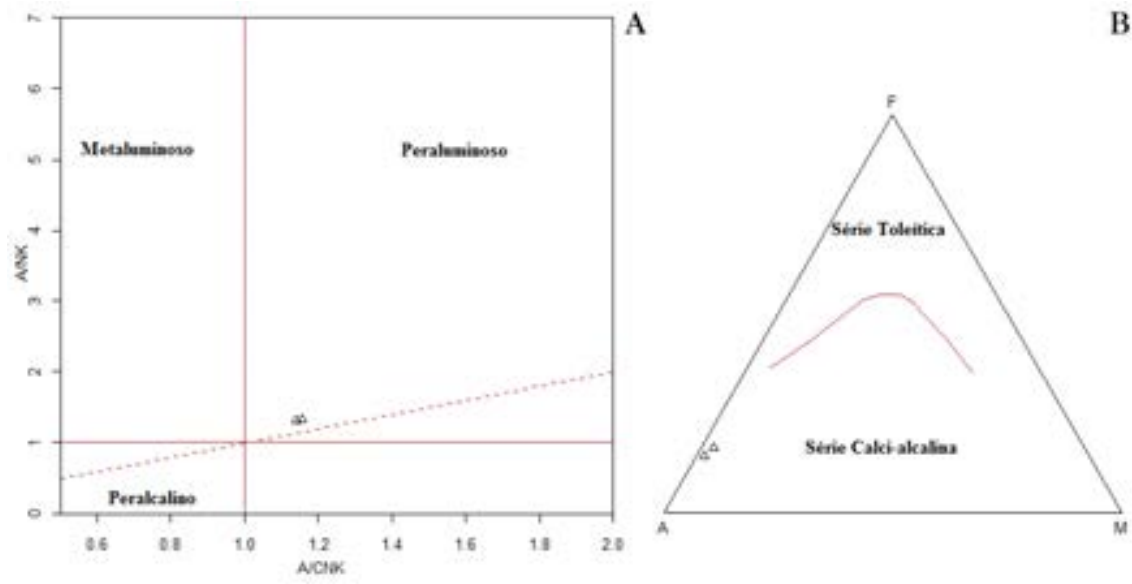

Fig. 4 - A: Diagramas de SHAND (1943) e B: AFM de IRVINE \& BARAGAR (1971) para o granito de Inchope. 
Tabela 1 - Resultados das análises químicas de elementos maiores.

\begin{tabular}{ccccccccccccc}
\hline & $\mathrm{SiO}_{2}$ & $\mathrm{TiO}_{2}$ & $\mathrm{Al}_{2} \mathrm{O}_{3}$ & $\mathrm{Fe}_{2} \mathrm{O}_{3}$ & $\mathrm{MnO}$ & $\mathrm{MgO}$ & $\mathrm{CaO}$ & $\mathrm{Na}_{2} \mathrm{O}$ & $\mathrm{K}_{2} \mathrm{O}$ & $\mathrm{P}_{2} \mathrm{O}_{5}$ & LOI & Total \\
\hline $\mathrm{VM} 015$ & 72,41 & 0,248 & 14,38 & 1,58 & 0,024 & 0,2 & 0,87 & 3,04 & 5,57 & 0,199 & 0,66 & 99,18 \\
$\mathrm{VM} 025$ & 72,92 & 0,253 & 14,14 & 1,83 & 0,04 & 0,29 & 0,9 & 3,01 & 5,23 & 0,25 & 0,6 & 99,46 \\
\hline
\end{tabular}

Para entender o padrão de abundância de elementos traço em granitóides usou-se o diagrama spider de SUN et al. (1980), no qual as abundâncias dos elementos incompatíveis são normalizadas para os condritos. Neste diagrama, os picos máximo e mínimo, as curvaturas e os declives dos padróes podem fornecer informaçóes petrogenéticas relativos ao equilíbrio cristal-líquido (WILSON, 1989). Todas a rochas mostram anomalias negativas de $\mathrm{Nb}$ e $\mathrm{Ti}$ (Fig. 5), sugerindo ambiente cálcio-alcalino de arco de ilha ou envolvimento de material crustal nos processos magmáticos. Mostram, ainda, anomalia negativa de $\mathrm{Sr}$, que provavelmente resulta da cristalização fraccional da plagioclase.

Os espectros de elementos de terras raras mostram, no geral, um forte enriquecimento em ETRL em comparação aos ETRP (Fig. 5 e tabela 2). Isto pode indicar a presença de granada, zircão ou hornblenda na fonte. Observa-se uma expressiva anomalia negativa de $\mathrm{Eu}$, que pode significar remoção de feldspato da fusão félsica por fracionamento do cristal.
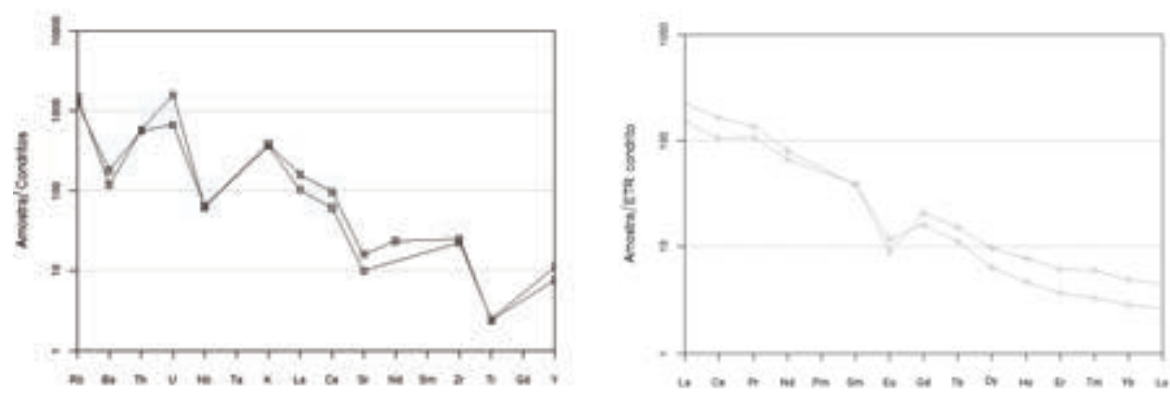

Fig. 5 - Diagrama spider (à esquerda) normalizado em relação ao Condrito (SUN et al., 1980). Diagrama de elementos de terras raras (à direita) normalizados segundo o Condrito de NAKAMURA (1974).

Tabela 2 - Valores medidos de elementos terras raras.

\begin{tabular}{ccccccccccccccc}
\hline & $\mathrm{La}$ & $\mathrm{Ce}$ & $\mathrm{Pr}$ & $\mathrm{Nd}$ & $\mathrm{Sm}$ & $\mathrm{Eu}$ & $\mathrm{Gd}$ & $\mathrm{Tb}$ & $\mathrm{Dy}$ & $\mathrm{Ho}$ & $\mathrm{Er}$ & $\mathrm{Tm}$ & $\mathrm{Yb}$ & $\mathrm{Lu}$ \\
\hline VMO15 & 74,7 & 143 & 15,5 & 51,3 & 7,86 & 0,9 & 4,5 & 0,53 & 2,19 & 0,33 & 0,83 & 0,1 & 0,62 & 0,09 \\
VMO25 & 50,8 & 91,2 & 12,2 & 42,4 & 7,98 & 0,69 & 5,79 & 0,73 & 3,31 & 0,54 & 1,37 & 0,18 & 1,08 & 0,15 \\
\hline
\end{tabular}

Além do padrão comum de fracionamento, os espectros dos ETR das amostras da área de estudo mostram moderado grau de empobrecimento em Eu, indicado pelos valores calculados de Eu/Eu* (Tabela 3) que variam de 0,31 - 0,47 para o Granito de Inchope. 
Para a discriminação de ambientes tectônicos na área de estudo usou-se o diagrama de PEARCE et al. (1984). Segundo este diagrama, as amostras plotam no campo dos granitóides de arco vulcânico e sin-orogênicos.

Tabela 3 - Elementos terras raras normalizados para o Condrito segundo NAKAMURA (1974).

\begin{tabular}{cccccccc}
\hline Amostra & Rocha & $\mathrm{Eu} / \mathrm{Eu}^{*}$ & $\mathrm{La}_{\mathrm{N}} / \mathrm{Yb}_{\mathrm{N}}$ & $\mathrm{La}_{\mathrm{N}} / \mathrm{Sm}_{\mathrm{N}}$ & $\mathrm{Ce}_{\mathrm{N}} / \mathrm{Yb}_{\mathrm{N}}$ & $\mathrm{Ce}_{\mathrm{N}} / \mathrm{Sm}_{\mathrm{N}}$ & $\mathrm{Eu}_{\mathrm{N}} / \mathrm{Yb}_{\mathrm{N}}$ \\
\hline VMO15 & granito & 0,47 & 80,52 & 5,85 & 58,85 & 4,27 & 4,16 \\
VMO25 & granito & 0,31 & 31,49 & 3,92 & 21,55 & 2,68 & 1,83 \\
\hline
\end{tabular}

\section{3 - Geocronologia e Geoquímica Isotópica}

Os resultados obtidos foram plotados em diagramas de Wetherill que se baseiam numa curva concórdia. Nestes, usaram-se erros nos elipsóides de $1 \sigma$ a um intervalo de confiança de $95 \%$. Para o granito de Inchope, amostra VM011, foi obtida uma idade de $1065 \pm 13 \mathrm{Ma}$ interpretada como de cristalização. Esta idade corresponde a média das idades ${ }^{207} \mathrm{~Pb} /{ }^{206} \mathrm{~Pb}$ apresentada na Fig. 6A. Na amostra VM015 foram analisados vinte e sete (26) spots em vinte (20) grãos de zircão usando como linha de orientação as imagens de catodoluminiscência. Esta amostra forneceu idade de $1053 \pm 19$ Ma interpretada como evento de cristalização (Fig. 6B). Alguns pontos analíticos definiram ainda duas idades de $956 \pm 38$ Ma e 484,2 +8,7/-6,6 Ma, que podem ser interpretadas como eventos de retrabalhamento, no início e no final do Neoproterozoico.

$\mathbf{A}$

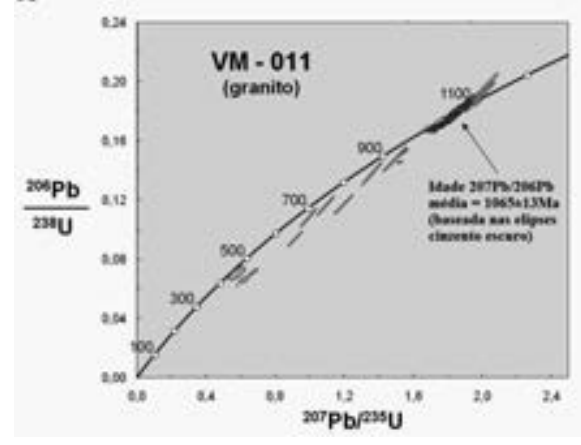

B

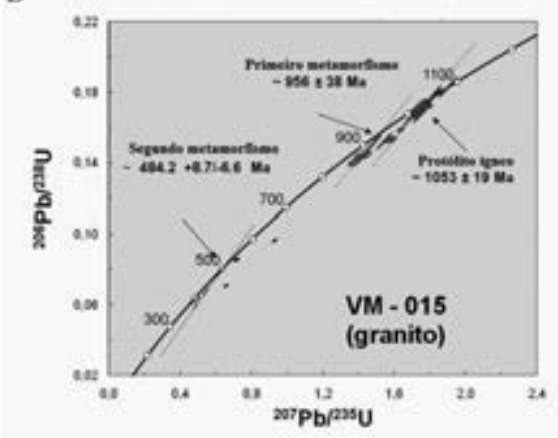

Fig. 6 - Diagramas concórdia U-Pb do Granito de Inchope.

Os resultados das análises isotópicas $\mathrm{Rb}-\mathrm{Sr}$ em rocha total e minerais são apresentados na tabela 4. O Granito de Inchope apresenta teores de Rb entre 438,6 e 561,2 ppm e de Sr entre 102,4 e $173,3 \mathrm{ppm}$. As razóes isotópicas ${ }^{87} \mathrm{Rb} /{ }^{86} \mathrm{Sr}$ variam entre 7,413 - 16,284 $\mathrm{e}^{87} \mathrm{Sr} /{ }^{86} \mathrm{Sr}$ entre $0,828486-0,953692$. Com estes dados foi possível calcular as idades isocrônicas em rocha total de $1026 \pm 82 \mathrm{Ma}$ e a razão inicial ${ }^{87} \mathrm{Sr} /{ }^{86} \mathrm{Sri}$ de $0,720 \pm 0,014$, bem como idade isocrônica em rocha total e minerais de $453 \pm 12 \mathrm{Ma}$ (Fig. 7). 
Tabela 4 - Dados isotópicos Rb-Sr para amostras da área de estudo.

\begin{tabular}{ccccccccc}
\hline SPR & Amostra & Material & $\mathrm{Rb}(\mathrm{ppm})$ & $\mathrm{Sr}(\mathrm{ppm})$ & ${ }^{87} \mathrm{Rb} /{ }^{86} \mathrm{Sr}$ & erro $(1 \sigma)$ & ${ }^{87} \mathrm{Sr} /{ }^{86} \mathrm{Sr}$ & erro $(2 \sigma)$ \\
\hline 6725 & VM025 & Granito & 561,2 & 102,2 & 16,284 & 0,061 & 0,953692 & 0,000066 \\
6726 & VM012 & Granito & 519,6 & 106,4 & 14,453 & 0,082 & 0,936048 & 0,000119 \\
6727 & VM015 & Granito & 438,6 & 173,3 & 7,413 & 0,063 & 0,828486 & 0,000064 \\
6728 & VM011 & Granito & 481,9 & 101,4 & 14,047 & 0,039 & 0,925961 & 0,000074 \\
16223 & VM011 & Musc./Granito & 1384,4 & 22,96 & 198,51 & 2,63 & 2,11563 & 0,001135 \\
16222 & VM011 & Biot./Granito & 2404,2 & 9,5 & 1356,310 & 17,197 & 9,484011 & 0,004233 \\
\hline
\end{tabular}
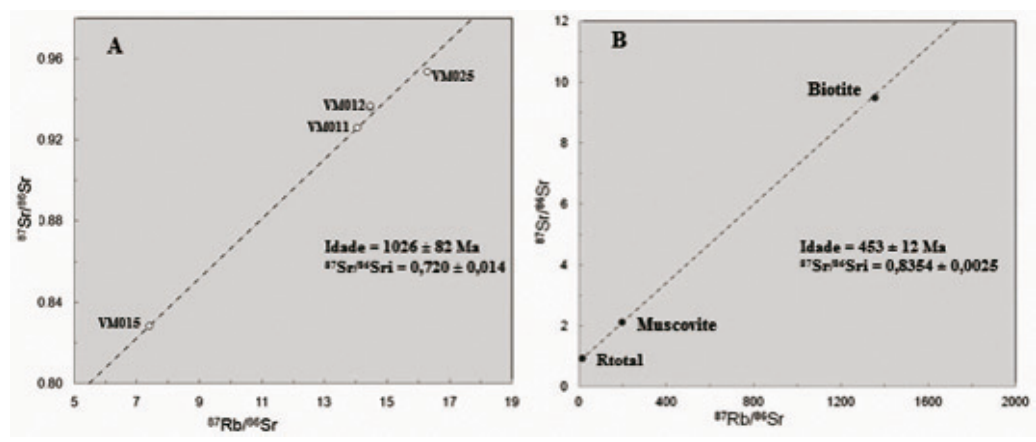

Fig. 7 - A: Diagramas isocrônicos Rb-Sr em rocha total do Granito de Inchope e B: em rocha total e minerais da amostra VM011.

O Granito de Inchope (tabela 5) apresenta teores de Sm entre 6,470 e 7,260 ppm e de $\mathrm{Nd}$ entre 34,075 e 41,908 ppm. As razóes isotópicas ${ }^{147} \mathrm{Sm} /{ }^{144} \mathrm{Nd}$ variam entre 0,0977 e 0,1148 e ${ }^{143} \mathrm{Nd} /{ }^{144} \mathrm{Nd}$ entre 0,511279 e 0,511474 . Tomando como base a razão ${ }^{143} \mathrm{Nd} /{ }^{144} \mathrm{Nd}$ de 0,512638 (CHUR) e ${ }^{87} \mathrm{Sr} /{ }^{86} \mathrm{Sr}$ de 0,7045 (UR) (bulk earth), foi possível verificar, através dos diagramas combinados $\mathrm{Sr}-\mathrm{Nd}$, que o Granito de Inchope tem fonte associada com alta razão $\mathrm{Rb} / \mathrm{Sr}$ e baixa razão $\mathrm{Sm} / \mathrm{Nd}$ comparativamente ao reservatório condrítico (CHUR). Tal fonte é chamada enriquecida.

Tabela 5 - Dados isotópicos Sm-Nd para amostras da área de estudo.

\begin{tabular}{cccccccccccc}
\hline Amostra & $\begin{array}{c}\mathrm{Sm} \\
(\mathrm{ppm})\end{array}$ & $\begin{array}{c}\mathrm{Nd} \\
(\mathrm{ppm})\end{array}$ & $\begin{array}{c}{ }^{147} \mathrm{Sm} / \\
{ }^{144} \mathrm{Nd}\end{array}$ & erro $^{1}$ & $\begin{array}{c}{ }^{143} \mathrm{Nd} / \\
{ }^{144} \mathrm{Nd}\end{array}$ & $\operatorname{erro}(2 \sigma)$ & $\mathrm{f}_{\mathrm{Sm} / \mathrm{Nd}}$ & $\begin{array}{c}\mathrm{T}_{\mathrm{DM}} \\
(\mathrm{Ga})\end{array}$ & $\varepsilon \mathrm{Nd}(\mathbf{0})$ & $\mathrm{T}(\mathrm{Ma})$ & $\varepsilon_{\mathrm{Nd}(\mathrm{T})}$ \\
\hline VM025 & 6,470 & 34,075 & 0,1148 & 0,0007 & 0,511474 & 0,000009 & $-0,42$ & 2,43 & $-22,70$ & 1026,0 & $-11,99$ \\
VM012 & 7,260 & 39,436 & 0,1113 & 0,0007 & 0,511457 & 0,000008 & $-0,43$ & 2,37 & $-23,05$ & 1026,0 & $-11,87$ \\
VM015 & 6,770 & 41,908 & 0,0977 & 0,0006 & 0,511279 & 0,000005 & $-0,50$ & 2,33 & $-26,51$ & 1026,0 & $-13,56$ \\
VM011 & 6,821 & 36,683 & 0,1124 & 0,0007 & 0,511429 & 0,000011 & $-0,43$ & 2,44 & $-23,58$ & 1026,0 & $-12,55$ \\
\hline
\end{tabular}

Os valores de $\varepsilon_{\mathrm{Nd}}$ no tempo de cristalização (definido pelas idades $\mathrm{Rb}-\mathrm{Sr}$ em rocha total) do Granito de Inchope variam de -11,87 a -13,56. Estes valores plotados contra ${ }^{87} \mathrm{Sr} /{ }^{86} \mathrm{Sr}$ indicam material da crosta superior como predominante no magma que gerou o Granito de Inchope. As idades modelo de manto empobrecido $\left(\mathrm{T}_{\mathrm{DM}}\right)$ mostram valores que variam de 2,33 a 2,44 para granitos de Inchope (tabela 5). Estes dados implicam que o Granito de Inchope originou-seda fusão parcial de rochas do Paleoproterozoico. 


\section{5 - Conclusóes}

O Granito de Inchope apresenta um carácter ácido, peraluminoso e cálcio-alcalino. Este granito possui origem híbrida, envolvendo material derivado do manto e magma produzido por processos de fusão parcial de rochas crustais. Ele mostra anomalias negativas de Nb e Ti nos diagramas de variação, sugerindo ambiente cálcio-alcalino de arco de ilha. Em adição, apresenta anomalia negativa de $\mathrm{Sr}$, que provavelmente resulta da cristalizaçáo fracionada da plagioclase.

Os espectros de elementos de terras raras mostram, no geral, duas características. A primeira é um forte enriquecimento em ETRL relativamente aos ETRP. Isto pode indicar a presença de granada, zircão ou hornblenda na fonte. Em adição, observa-se proeminente anomalia negativa de Eu. Isto pode significar fusão parcial de uma rocha na qual o feldspato é retido na fonte.

Segundo o diagrama de PEARCE et al. (1984), para a discriminação de ambientes tectônicos, o Granito de Inchope plota no campo dos granitóides de arco vulcânico e sin-orogênicos.

O Granito de Inchope apresenta idades de cristalização próximas a $1060 \mathrm{Ma}(1065 \pm$ $13 \mathrm{Ma}$ e $1053 \pm 13 \mathrm{Ma}$ ), obtidas pelo método U-Pb usando LA-ICP-MS em zircôes nas amostras VM011 e VM015, respectivamente. Estas idades estão em concordância com a obtida na mesma unidade litológica por U-Pb usando SHRIMP em zircôes [1079 \pm 7 Ma em metagranodiorito e $1119 \pm 21$ Ma no granito; GTK CONSORTIUM (2006) e MANTTARI (2008)] e por Rb-Sr em rocha total de $1026 \pm 82 \mathrm{Ma}$ (presente estudo). Este granito sofreu a ação de eventos tectono-magmáticos relacionados com o Neoproterozoico, conforme as idades U-Pb em zircão mais jovens, de $958 \pm 38 \mathrm{Ma}$ e 484,2 $+8,7 /-6,6 \mathrm{Ma}$ na amostra VM011 e Rb-Sr em rocha total e micas de 453 $\pm 12 \mathrm{Ma}$. Estas idades são concordantes com as obtidas em monazite por TIMS (530-520 Ma; GTK CONSORTIUM, 2006). MANHIÇA et al. (2001) e GRANTHAM et al. (2011) reportam idades de cristalizaçáo similares às obtidas no presente estudo (1139 Ma para o Granodiorito de Chimoio) confirmando uma granitogênese Grenviliana ou Kibariana na região. Estes granitóides se correlacionam com os do Cinturão Orogênico Kibariano com idades de cristalização de $986 \pm 10 \mathrm{Ma}$ e mineralizaçóes Sn-W-Ta (DEWAELE et al., 2011).

Os valores de $\varepsilon_{\mathrm{Nd}}$ calculados para época de cristalização variam de -11 a -13 e são consideravelmente inferiores à média do manto empobrecido (+ 4; DE PAOLO 1981), indicando a participação de rochas crustais nos respectivos magmas parentais, e podem significar uma associação com arcos em margens continentais. Os valores de $\varepsilon_{\mathrm{Nd}}$ versus razão inicial de ${ }^{87} \mathrm{Sr} /{ }^{86} \mathrm{Sr}$ indicam material da crusta superior como composição predominante do magma que gerou o Granito de Inchope $\left(\varepsilon_{\mathrm{Nd}}=-11,87\right.$ a $\left.-13,56 \mathrm{e}^{87} \mathrm{Sr} /{ }^{86} \mathrm{Sri}=0,720\right)$. Os dados isotópicos sugerem que o Granito de Inchope foi gerado por fusão parcial envolvendo mistura (valores negativos de $\varepsilon_{\mathrm{Nd}}$ ) do material da crusta Arcaica/Paleoproterozoica e magma Mesoproterozoico a 1100 Ma.

Agradecimentos - Ao Conselho Nacional de Desenvolvimento Científico e Tecnológico - CNPq que financiou a realização das análises. 


\section{Referências Bibliográficas}

DE PAOLO, D. J., LINN, A. M. \& SCHUBBERT, G. (1991) - The continental age distribution: methods of determining mantle separation ages from $\mathrm{Sm}-\mathrm{Nd}$ isotopic data and application to the southwestern United States. Journal of Geophysical Research, 96, p. 2071-2088.

DEWAELE, S., HENJES-KUNST, F., MELCHER, F., SITNIKOVA, M., BURGESS, R., GERDES, A., FERNANDES, M. A., CLERCQ, F. D., MUCHEZ, P., \& LEHMAN, N. (2011) - Late Neoproterozoic overprinting of the cassiterite and columbite-tantalite bearing pegmatites of the Gatumba area, Rwanda (Central Africa). Journal of African Earth Sciences, 61, p. 10-26.

GRANTHAM, G. H., MANHIÇA, A. D., ARMSTRONG, R. A., KRUGER, F. J. \& LOUBSER, M. (2011) - New SHRIMP, Rb/Sr and Sm/Nd isotope and whole rock chemical data from central Mozambique and western Dronning Maud Land, Antarctica: Implications for the nature of the eastern margin of the Kalahari Craton and the amalgamation of Gondwana. Journal of African Earth Sciences, 59, p. 74-100.

GTK CONSORTIUM (2006) - Geology of Degree Sheets Mecumbura, Chioco, Tete, Tambara, Guro, Chemba, Manica, Catandica, Gorongosa, Rotanda, Chimoio and Beira. Geological Mapping. Ministério dos Recursos Minerais, Direcção Nacional de Geologia. p. 411. http://www.cenacarta.com/modules. php?name=Downloads\&d_op=getit\&lid=6. Acessado em 6 de Fevereiro de 2012.

IRVINE, T. N. \& BARAGAR, W. R. (1971) - A guide to the chemical classification of the common volcanic rocks. Canadian Journal of Earth Sciences, 8, p. 523-548.

KORKIAKOSKI, E. (2007) - Component 2: Geological Infrastructure Development Programme; Geochemical and Industrial Mineral Surveys (GIM). FINAL REPORT, Ministério dos Recursos Minerais, Direcção Nacional de Geologia, Maputo.

LACHELT, S. (2004) - Geology and Mineral Resources of Mozambique (Vol. II). Maputo: Council For Geoscience, p. 205.

LKAB (1980) - Trabalho de Prospeção preliminar na área de Inchope-Doeroi, Moçamique. Ministério dos Recursos Minarais, Direção Nacional de Geologia. Maputo: LKAB INTERNATIONAL AB.

MANHiÇA, A. D., GRAntham, G., ARMSTrOnG, R. A., GUiSE, P. G. \& KRUGER, F. J. (2001) - Polyphase deformation and metamorphism at the Kalahari Craton - Mozambique Belt boundary. Miller, J. A., Holdsworth, R. E., Buick, I. S. \& Hand, M. (eds.) Geological Society. Special publication, 184, p. 303-322.

MANNINEN, T., EEROLA, T., MAKITIE, H., VUORI, S., LUTTINEN, A., SÉNVANO, A., \& MANHIÇA, V. (2008) - The karoo volcanic rocks and related intrusions in southern and central Mozambique. Geological Survey of Finland, Special Paper, 48, p. 211-250.

MANTTARI, I. (2008). Mesoarchaean to lower jurassic U-Pb and Sm-Nd ages from NW Mozambique. Geological Survey of Finland, Special Paper, 48, p. 81-119.

MELlUSO, L., CENSI, P., PERINI, G., VASCONCELOS, L., MORRA, V., GUERREIRO, F., \& BENNIO, L. (2004) - Chemical and isotopic (C, O, Sr, Nd) characteristics of the Xiluvo carbonatite (central-western Mozambique). (L. Gwalani, Ed.) Mineralogy and Petrology, 80, p. 201-213.

NAKAMURA, N. (1974) - Determination of REE, Ba, Fe, Mg, Na and K in carbonaceous and ordinary chondrites. Geochim Cosmochim Acta, 38, p. 757-775.

PEARCE, J. A., HARRIS, N. W. \& TINDLE, A. G. (1984) - Trace element discrimination diagrams for the tectonic interpretation of granitic rocks. J Petrology, 25, p. 956-983.

PINNA, P., MARTEAU, P., BECQ-GIRAUDON, J-F. \& MANIGAULT, B. (1987) - Carta Geológica de Moçambique, na escala 1:1 000 000. Instituto Nacional de Geologia de Moçambique, Maputo.

SHAND, S. J. (1943) - Eruptive Rocks: Their genesis, composition, classification, and their relation to ore-deposits with a chapter on meteorites, p. 444.

SUN, S. S., BAILEY, D. K., TARNEY, J. \& DUNHAM, K. (1980) - Lead isotopic study of young volcanic rocks from mid-ocean ridges, ocean islands and island arcs. Philos Trans $R$ Soc London, A297, p. 409-445.

WILSON, M. (1989) - Igneous Petrogenesis: A global tectonic approach. London. Chapman \& Hall, p. 466. 